Volume 25 (2019) 1-11

DOI: $10.24330 /$ ieja.504095

\title{
LOCAL COMPARABILITY OF EXCHANGE IDEALS
}

\author{
Handan Kose, Yosum Kurtulmaz and Huanyin Chen \\ Received: 26 December 2017; Revised: 7 September 2018; Accepted: 18 October 2018 \\ Communicated by W. Keith Nicholson
}

\begin{abstract}
An exchange ideal $I$ of a ring $R$ is locally comparable if for every regular $x \in I$ there exists a right or left invertible $u \in 1+I$ such that $x=x u x$. We prove that every matrix extension of an exchange locally comparable ideal is locally comparable. We thereby prove that every square regular matrix over such ideal admits a diagonal reduction.
\end{abstract}

Mathematics Subject Classification (2010): 16S34, 16E50, 16U99, 13 B99

Keywords: Locally comparable ideal, matrix extension, diagonal reduction, exchange ideal

\section{Introduction}

An element $x$ of a ring $R$ is regular if there exists $y \in R$ such that $x=x y x$. If, in addition, $y$ is right or left invertible, $x \in R$ is one-sided unit-regular. A ring $R$ is one-sided unit-regular provided that every element in $R$ is one-sided unitregular. As is well known, a ring $R$ is one-sided unit-regular if and only if for all finitely generated projective right $R$-modules $A, B$ and $C, A \oplus B \cong A \oplus C$ implies that $B \lesssim^{\oplus} C$ or $C \lesssim^{\oplus} B$ (see [2] and [5]). In [2], Chen proved that comparability of modules over one-sided unit-regular rings is Morita invariant, in terms of comparability. In [3], the author considered a class of ideals in a regular ring. In [4], the author introduced and investigated a kind of quasi-stable exchange ideals. These inspires us to explore local comparability depending only on the ring structure of an ideal and then investigate certain matrix reduction over rings which might have no any comparability.

Following Ara, an ideal $I$ of a ring $R$ is an exchange ideal provided that for every $x \in I$ there exist an idempotent $e \in I$ and elements $r, s \in I$ such that $e=x r=x+s-x s$ (cf. [1]). Many classes of ideals of interest belong to such one, e.g., regular ideals, $\pi$-regular ideals ([5] and [9]).

Huanyin Chen was supported by the Natural Science Foundation of Zhejiang Province, China (No. LY17A010018). 
Let $I$ be an ideal of a ring $R$, let $U^{-}(R)$ be the set of all right or left invertible elements in $R$, and let $U^{-}(I)=U^{-}(R) \cap(1+I)$. We call an ideal $I$ of a ring $R$ is locally comparable if for each regular element $x \in I$ there exists $u \in U^{-}(I)$ such that $x=x u x$. For instance, every ideal of a commutative ring and every ideal of a unit-regular ring. Following Khurana, Lam and Nielsen in [7], a ring $R$ is IC provided that every regular element in $R$ is unit-regular. Thus, every ideal of an IC ring is locally comparable. We prove that every matrix extension of an exchange locally comparable ideal is locally comparable. From this, we show that every square regular matrix over such ideal admits a diagonal reduction.

Throughout, all rings are associative with identity and all modules are right modules. We use $M_{n}(I)$ to denote the set of $n \times n$ matrices over an ideal $I$ and $G L_{n}(R)$ to denote the $n$ dimensional general linear group of $R . \quad M \lesssim^{\oplus} N$ means that $M$ is isomorphic to a direct summand of $N$.

\section{Locally comparable ideals}

For further use, we now investigate when an exchange ideal of a ring is locally comparable which will be frequently used. We begin with

Lemma 2.1. Let $I$ be an ideal of a ring $R$. Then the following are equivalent:

(1) I is an exchange ideal.

(2) For any $x \in I$, there exists an idempotent $e \in x R$ such that $1-e \in(1-x) R$.

(3) For any $x \in 1+I$, there exists an idempotent $e \in x R$ such that $1-e \in$ $(1-x) R$.

Proof. Straightforward.

Theorem 2.2. Let $I$ be an exchange ideal of a ring $R$. Then the following are equivalent:

(1) I is locally comparable.

(2) Whenever $a R+b R=R$ with $a \in I, b \in R$, there exists $y \in R$ such that $a+b y \in U^{-}(I)$.

Proof. (1) $\Rightarrow(2)$ Suppose that $a R+b R=R$ with $a \in I, b \in R$. Then we have $x, y \in R$ such that $a x+b y=1$. Since $I$ is an exchange ideal and $b y=1-a x \in 1+I$, we have an idempotent $e \in$ by $R$ such that $1-e \in(1-b y) R$ by Lemma 2.1. So $e=$ bys and $1-e=a x t$ for some $s, t \in R$. Hence $(1-e) \operatorname{axt}(1-e)+e=1$, and then $(1-e) a \in I$ is regular. As $I$ is locally comparable, we have $u \in U^{-}(I)$ such that 
$(1-e) a=(1-e) a u(1-e) a$. Set $f=u(1-e) a$. Then $f(x t(1-e)+u e)+(1-f) u e=u$. If $v u=1$ for some $v \in R$,

$$
(1-f) u e v(1-f) u e=(1-f) u e(e-(1-e) a u e)=(1-f) u e .
$$

If $u v=1$ for some $v \in R$,

$$
(1-f) u e v(1-f) u e=(1-f)(1-f x t(1-e))(1-f) u e=(1-f) u e .
$$

Let $g=(1-f) u e v(1-f)$. Then $f(x t(1-e)+u e)+g u e=u$. Clearly, $f(x t(1-$ $e)+u e)=f u$ and $g u e=g u$. Thus

$$
\begin{aligned}
& {[u(a+\operatorname{bys}(v(1-f)(1+f u e v(1-f))-a))](1-f u e v(1-f)) u} \\
& =[f+u e v(1-f)(1+f u e v(1-f))](1-f u e v(1-f)) u \\
& =u
\end{aligned}
$$

As $u \in U^{-}(I)$ and $1-f u e v(1-f) \in 1+I$, we get $a+b z \in U^{-}(I)$, where $z=y s(v(1-f)(1+f u e v(1-f))-a)$.

$(2) \Rightarrow(1)$ Given any regular $x \in I$, we have $y \in R$ such that $x=x y x$. Hence $x=x z x$ and $z=y x y \in I$. From $z R+(1-z x) R=R$ with $z \in I$, we can find $s \in R$ such that $z+(1-z x) s=u \in U^{-}(I)$. Therefore $x=x z x=x(z+(1-z x)) x=x u x$, as required.

Corollary 2.3. Let $I$ be an exchange ideal of $R$. Then the following are equivalent:

(1) I is locally comparable.

(2) For any regular $a, b \in I, a R=b R$ implies that there exists $u \in U^{-}(I)$ such that $a=b u$.

(3) For any regular $a, b \in I, R a=R b$ implies that there exists $u \in U^{-}(I)$ such that $a=u b$.

Proof. (1) $\Rightarrow(2)$ Given $a R=b R$ with regular $a, b \in I$, then $a=b x, b=a y$ for $x, y \in R$. Since $b$ is regular, there exists $c \in R$ such that $b=b c b$. Thus $a=b(c b x)$ and $b=a y$; hence, $b=b(c b x) y$. As $(c b x) y+(1-c b x y)=1$ with $c b x \in I$, by Theorem 2.2, we can find $z \in R$ such that $c b x+(1-c b x y) z=u \in U^{-}(I)$. This infers that $a=b x=b(c b x)=b(c b x+(1-c b x y) z)=b u$.

$(2) \Rightarrow(1)$ Given any regular $x \in I$, we have $y \in I$ such that $x=x y x$. Set $e=y x$. Then $e=e^{2} \in I$. As $y R=e R$, we have $y=e v$ for a $v \in U^{-}(I)$. From $y x+(1-y x)=1$, we see that eux $+(1-y x)=1$; hence,

$$
y+(1-y x)(1-e) v=(1+\operatorname{evx}(1-e))^{-1} u .
$$

Set $u=(1+\operatorname{eux}(1-e))^{-1} v$. Clearly, $(1+\operatorname{eux}(1-e))^{-1} \in 1+I$. Thus, $x=x y x=$ $x u x$ and $u \in U^{-}(I)$, as required. 
$(1) \Leftrightarrow(3)$ Since $I$ is locally comparable in $R$ if and only if $I^{o p}$ is locally comparable of the opposite ring $R^{o p}$, we complete the proof by applying $(1) \Leftrightarrow(2)$ to the ideal $I^{o p}$ of $R^{o p}$.

Corollary 2.4. Let $I$ be an exchange locally comparable ideal of a ring $R$. Then for any regular $a, b \in I, a R \cong b R$ implies that $b=$ uav for some $u, v \in U^{-}(I)$.

Proof. It is clear from Corollary 2.3.

The following result shows that the local comparability only depends on the ring structure of an ideal.

Corollary 2.5. Let $I$ be an ideal of a ring $R$. Then the following are equivalent:

(1) I is locally comparable.

(2) Whenever $a x+b=1$ with $a, x \in I, b \in I$, there exists $y \in 1+I$ such that $a+b y \in U^{-}(I)$.

Proof. $(1) \Rightarrow(2)$ is clear by Theorem 2.2.

$(2) \Rightarrow(1)$ Given any regular $x \in I$, there exists $y \in I$ such that $x=x y x$ and $y=y x y$. From $y x+(1-y x)=1$, we have $z \in 1+I$ such that $y+(1-y x) z=u \in$ $U^{-}(I)$. Therefore $x=x y x=x(y+(1-y x) z) x=x u x$, as required.

Example 2.6. Let $V$ be an infinite dimensional vector space over a division ring $D$. Let $R=\mathbb{Z} \oplus \operatorname{End}_{D}(V)$. Then $I=0 \oplus \operatorname{End}_{D}(V)$ is a locally comparable ideal of $R$.

Example 2.7. Let $R=\left\{\left(\begin{array}{ll}a & b \\ 0 & a\end{array}\right) \mid a, b \in \mathbb{Z}\right\}$. Then $I=\left\{\left(\begin{array}{ll}a & b \\ 0 & a\end{array}\right) \mid a, b \in\right.$ $\mathbb{Z}, 2 \mid a\}$ is a locally comparable ideal of $R$.

\section{Matrix extensions}

The pair $(a, b)$ is called an $I$-unimodular row in case $a x+b y=1$ for some $x \in I, y \in R$. The $I$-unimodular row $(a, b)$ is called weakly $I$-reducible if there exists $z \in R$ such that $a+b z \in U^{-}(I)$. In [5], Chen proved that if $R$ is one-sided unit-regular then so is $M_{n}(R)$ by virtue of comparability of $R$-modules. The goal of this section is to prove that local comparability is inhered by matrix extensions.

Lemma 3.1. Let $(a, b)$ be an $I$-unimodular row in a ring $R$. Let $u, v \in U(R) \cap(1+I)$ and $c \in R$. Then $(v a u+v b c, v b)$ is also an I-unimodular row, and that $(a, b)$ is weakly I-reducible if and only if so is $(v a u+v b c, v b)$. 
Proof. Straightforward.

Following the similar route, we shall modify the proofs of [3, Theorem 3.2] and [4, Theorem 3.2] to our case. We are ready to prove:

Theorem 3.2. Let $I$ be an exchange locally comparable ideal of a ring $R$. Then $M_{n}(I)$ is an exchange locally comparable ideal of $M_{n}(R)$.

Proof. The result holds for $n=1$. We now induct on $n$. Assume that the result holds for $n$. It will suffice to show that the result holds for $n+1$. Suppose that $\left(a_{i j}\right)\left(b_{i j}\right)+\left(c_{i j}\right)=I_{n+1}(*)$ in $M_{n+1}(R)$, where $\left(a_{i j}\right),\left(b_{i j}\right) \in M_{n+1}(I)$. Then

$$
a_{11} b_{11}+a_{12} b_{21}+\cdots+a_{1(n+1)} b_{(n+1) 1}+c_{11}=1
$$

with $a_{11} \in I$. Since $I$ is an exchange locally comparable ideal of $R$, by Theorem 2.2 , there exists $z_{1} \in R$ such that

$$
a_{11}+\left(a_{12} b_{21}+\cdots+a_{1 n} b_{n 1}+c_{11}\right) z_{1} \in U^{-}(I)
$$

It is easy to verify that $\left(\begin{array}{ccccc}1 & 0 & 0 & \cdots & 0 \\ b_{21} z_{1} & 1 & 0 & \cdots & 0 \\ b_{31} z_{1} & 0 & 1 & \cdots & 0 \\ \vdots & \vdots & \vdots & \ddots & \vdots \\ b_{(n+1) 1} z_{1} & 0 & 0 & \cdots & 1\end{array}\right) \in U\left(M_{n+1}(I)\right)$. According

to Lemma 3.1, (*) is weakly $M_{n+1}(I)$-reducible if and only if this is so for the $M_{n+1}(I)$-unimodular row with elements

$$
\left(a_{i j}\right)_{n+1}\left(\begin{array}{ccccc}
1 & 0 & 0 & \cdots & 0 \\
b_{21} z_{1} & 1 & 0 & \cdots & 0 \\
b_{31} z_{1} & 0 & 1 & \cdots & 0 \\
\vdots & \vdots & \vdots & \ddots & \vdots \\
b_{(n+1) 1} z_{1} & 0 & 0 & \cdots & 1
\end{array}\right)+\left(c_{i j}\right)_{n+1}\left(\begin{array}{ccccc}
z_{1} & 0 & 0 & \cdots & 0 \\
0 & 0 & 0 & \cdots & 0 \\
0 & 0 & 0 & \cdots & 0 \\
\vdots & \vdots & \vdots & \ddots & \vdots \\
0 & 0 & 0 & \cdots & 0
\end{array}\right)
$$

and $\left(c_{i j}\right)$. Thus we may assume that the element $a_{11} \in U^{-}(I)$. Obviously, $c_{21}, \cdots, c_{(n+1) 1} \in I$. Hence $a_{i j} \in I$ (either $i \neq 1$ or $j \neq 1$ ) in $(*)$, and then we have $s, t \in R$ such that $s a_{11} t=1$, where $s=1$ or $t=1$. Clearly, $s, t \in 1+I$, and 
that

$$
\begin{aligned}
& \left(\begin{array}{ccccc}
s & 0 & 0 & \cdots & 0 \\
1-a_{11} t s & a_{11} t & 0 & \cdots & 0 \\
0 & 0 & 1 & \cdots & 0 \\
\vdots & \vdots & \vdots & \ddots & \vdots \\
0 & 0 & 0 & \cdots & 1
\end{array}\right) \\
& 0
\end{aligned}
$$

Similarly to [3, Theorem 3.2], we claim that $(*)$ is weakly $M_{n+1}(I)$-reducible if and only if this is so for the $M_{n+1}(I)$-unimodular row with elements

$$
\begin{gathered}
\left(\begin{array}{ccccc}
1 & d_{12} & d_{13} & \cdots & d_{1(n+1)} \\
d_{21} & d_{22} & d_{23} & \cdots & d_{2(n+1)} \\
d_{31} & d_{32} & * & \cdots & * \\
\vdots & \vdots & \vdots & \ddots & \vdots \\
d_{(n+1) 1} & d_{3(n+1)} & * & \cdots & d_{(n+1)(n+1)}
\end{array}\right) \\
\left(\begin{array}{ccccc}
s & 0 & 0 & \cdots & 0 \\
1-a_{11} t s & a_{11} t & 0 & \cdots & 0 \\
0 & 0 & 1 & \cdots & 0 \\
\vdots & \vdots & \vdots & \ddots & \vdots \\
0 & 0 & 0 & \cdots & 1
\end{array}\right)\left(c_{i j}\right)
\end{gathered}
$$

We easily see that $b_{i j} \in I$ (either $3 \leq i \leq n+1$ or $3 \leq j \leq n+1$ ) and $b_{12}=$ $s a_{11}\left(1-t s a_{11}\right)+s a_{12} s a_{11}, b_{21}=\left(1-a_{11} t s\right) a_{11} t+a_{11} t a_{21} t, b_{22}=\left(\left(1-a_{11} t s\right) a_{11}+\right.$ $\left.a_{11} t a_{21}\right)\left(1-t s a_{11}\right)+\left(\left(1-a_{11} t s\right) a_{12}+a_{11} t a_{22}\right) s a_{11} \in I$. By Lemma 3.1 again, we may assume that $a_{11}=1, a_{1 i}=0=a_{i 1}(2 \leq i \leq n+1)$ in $(*)$. Furthermore, we may assume that $(*)$ is in the following form:

$$
\begin{aligned}
& \left(\begin{array}{cc}
1 & 0_{1 \times n} \\
0_{n \times 1} & D
\end{array}\right)\left(\begin{array}{ll}
e_{11} & E_{12} \\
E_{21} & E_{22}
\end{array}\right)+\left(\begin{array}{ll}
c_{11} & C_{12} \\
C_{21} & C_{22}
\end{array}\right) \\
& =\left(\begin{array}{cc}
1 & 0 \\
0 & \operatorname{diag}(1, \cdots, 1)_{n}
\end{array}\right),
\end{aligned}
$$


$D \in M_{n}(I)$ and $\left(\begin{array}{cc}e_{11} & E_{12} \\ E_{21} & E_{22}\end{array}\right) \in M_{n+1}(I)$. Hence $D E_{22}+C_{22}=\operatorname{diag}(1, \cdots, 1)_{n}$.

By hypothesis, $M_{n}(I)$ is an exchange locally comparable, so we have $Z_{2} \in M_{n}(R)$ such that $D+C_{22} Z_{2} \in U^{-}\left(M_{n}(I)\right)$. As in [3, Theorem 3.2], we may pass to the $M_{n+1}(I)$-unimodular row with elements

$$
\left(\begin{array}{cc}
1 & 0_{1 \times n} \\
0_{n \times 1} & D
\end{array}\right)+\left(\begin{array}{cc}
c_{11} & C_{12} \\
C_{21} & C_{22}
\end{array}\right)\left(\begin{array}{cc}
0 & 0_{1 \times n} \\
0_{n \times 1} & Z_{2}
\end{array}\right),\left(\begin{array}{cc}
c_{11} & C_{12} \\
C_{21} & C_{22}
\end{array}\right) .
$$

In addition, we have $C_{12} \in M_{1 \times n}(I)$. It suffices to prove that $M_{n+1}(I)$-unimodular row with elements

$$
\left(\begin{array}{cc}
1 & C_{12} Z_{2} \\
0_{n \times 1} & D+C_{22} Z_{2}
\end{array}\right) \text { and }\left(\begin{array}{cc}
c_{11} & C_{12} \\
C_{21} & C_{22}
\end{array}\right)
$$

is weakly $M_{n+1}(I)$-reducible. As $D+C_{22} Z_{2} \in U^{-}\left(M_{n}(I)\right)$ and $C_{12} \in M_{1 \times n}(I)$, we conclude that $\left(\begin{array}{cc}1 & C_{12} Z_{2} \\ 0 & D+C_{22} Z_{2}\end{array}\right) \in U^{-}\left(M_{n+1}(I)\right)$. By induction, we complete the proof.

Lemma 3.3. (see [5, Lemma 13.1.4]) Let I be an exchange ideal of a ring $R$. Then $e$ Re is an exchange ring for all idempotents $e \in I$.

Lemma 3.4. (see [5, Lemma 13.1.8]) Let $I$ be an exchange ideal of a ring $R$. If $P$ is a finitely generated projective right $R$-module such that $P=P I$. Then there exist idempotents $e_{1}, \ldots, e_{n} \in I$ such that $P \cong e_{1} R \oplus \cdots \oplus e_{n} R$.

Theorem 3.5. Let $I$ be an exchange locally comparable ideal of a ring $R$. Then every square regular matrix over $I$ admits a diagonal reduction by right or left invertible matrices.

Proof. Given any regular $A \in M_{n}(I)$, then we have an idempotent matrix $E \in$ $M_{n}(I)$ such that $A M_{n}(R)=E M_{n}(R)$. Clearly, $E R^{n}$ is a finitely generated projective right $R$-module such that $E R^{n}=E R^{n} I$. Using Lemma 3.4, we have idempotents $e_{1}, \cdots, e_{n} \in I$ such that $E R^{n} \cong e_{1} R \oplus \cdots \oplus e_{n} R \cong \operatorname{diag}\left(e_{1}, \cdots, e_{n}\right) R^{n}$ as right $R$-modules, so $A M_{n}(R)=E M_{n}(R) \cong \operatorname{diag}\left(e_{1}, \cdots, e_{n}\right) M_{n}(R)$. Using Theorem 3.2 and [1, Theorem 1.4], $M_{n}(I)$ is an exchange locally comparable ideal of $M_{n}(R)$. In view of Corollary 2.4, there are $U, V \in U^{-}\left(M_{n}(I)\right)$ such that $U A V=\operatorname{diag}\left(e_{1}, \cdots, e_{n}\right)$, as asserted.

Corollary 3.6. Let $R$ be an exchange ring in which every regular element is onesided unit-regular. Then every square regular matrix over $R$ admits a diagonal reduction with idempotent entries by right or left invertible matrices. 
Proof. It is clear by Theorem 3.5.

Corollary 3.7. Let $R$ be one-sided unit-regular. Then every square matrix over $R$ admits a diagonal reduction with idempotent entries.

Proof. This is obvious from Corollary 3.6.

Let $V$ be an infinite dimensional vector space over a division $\operatorname{ring} D$. Set $R=$ $\operatorname{End}_{D}(V)$. Then $R$ is one-sided unit-regular. As a consequence of Corollary 3.7, we deduce that every row-column-finite matrices over a division ring admits a diagonal reduction with idempotent entries.

\section{The comparability axiom}

Let $I$ be an ideal of a ring $R$. We say that $I$ satisfies the comparability axiom provided that for any idempotents $e, f \in I$, either $e R \lesssim^{\oplus} f R$ or $f R \lesssim^{\oplus} e R$. We prove, in this section, that every exchange ideal satisfying the comparability axiom is a locally comparable ideal.

Lemma 4.1. Let $I$ be an ideal of a ring $R$. Suppose that $a x+b=1$ with $a, x \in$ $1+I, b \in R$. Then the following are equivalent:

(1) There exists $y \in R$ such that $a+b y \in U^{-}(I)$.

(2) There exists $z \in R$ such that $x+z b \in U^{-}(I)$.

Proof. As in the proof of [5, Lemma 4.1.2], we easily obtain this result.

Lemma 4.2. Let $I$ be an ideal of a ring $R$. Suppose that $a x+b=1$ with $a \in$ $1+I, x \in I, b \in 1+I$. Then the following statements are equivalent:

(1) There exists $y \in I$ such that $a+b y \in U^{-}(I)$.

(2) There exists $z \in I$ such that $x+z b \in U^{-}(I)$.

Proof. $(1) \Rightarrow(2)$ Suppose that $a+b y \in U^{-}(I)$ for a $y \in R$. Then $y \in I$. Assume that $u(a+b y)=1$. We have $u \in 1+I$. Similar to [6, Lemma 1$]$, we get

$$
(x+(1-x y) u b)(a+y(1-x a))=1
$$

and $x+(1-x y) u b \in 1+I$. Assume that $(a+b y) u=1$, Then $u \in 1+I$; hence, it follows from $(a+y(1-x a))(x+(1-x y) u b)=1$ that $x+(1-x y) u b \in U^{-}(I)$, as required.

$(2) \Rightarrow(1)$ Suppose that there exists $z \in R$ such that $x+z b \in U^{-}(I)$. Then $z \in 1+I$. Assume that $v(x+z b)=1$. We have $v \in 1+I$. Similar to $[6$, Lemma 1], $(a+b v(1-z a))(x+(1-x a) z)=1$. Since $a+b v(1-z a) \in 1+I$, 
$a+b v(1-z a) \in U^{-}(I)$. Assume that $(x+z b) v=1$. Then $v \in 1+I$. From $(x+(1-x a) z)(a+b v(1-z a))=1$, we deduce that $a+b v(1-z a) \in U^{-}(I)$, as asserted.

Lemma 4.3. Let $I$ be an exchange ideal of a ring $R$. Suppose that for any regular $x \in 1+I$ there exists $u \in U^{-}(I)$ such that $x=x u x$. Then $a x+b=1$ with $a \in 1+I, x \in I, b \in 1+I$ implies that $a+b y \in U^{-}(I)$ for any $y \in I$.

Proof. Suppose that $a x+b=1$ with $a \in 1+I, x \in I, b \in 1+I$. Then $a(x+b)+$ $(1-a) b=1$ with $a, x+b \in 1+I$. As $(1-a) b=1-a(x+b) \in I$ and $I$ is an exchange ideal of $R$, we have an idempotent $e \in R$ such that $e \in(1-a) b R$ and $1-e \in(1-(1-a) b) R$. So $e=(1-a) b s$ and $1-e=a(x+b) t$ for some $s, t \in R$. Hence $(1-e) a(x+b) t(1-e)+e=1$, and then $(1-e) a \in 1+I$ is regular. This infers that $(1-e) a=(1-e) a u(1-e) a$ for a $u \in U^{-}(R)$. Clearly, $u \in U^{-}(I)$. Let $f=u(1-e) a$. Then $f((x+b) t(1-e)+u e)+(1-f) u e=u$. If $v u=1$ for some $v \in R$, then

$$
(1-f) \text { uev }(1-f) \text { ue }=(1-f) \text { ue }(e-(1-e) \text { aue })=(1-f) \text { ue. }
$$

If $u v=1$ for some $v \in R$, then

$$
(1-f) u e v(1-f) u e=(1-f)(1-f(x+b) t(1-e) v)(1-f) u e=(1-f) u e .
$$

Let $g=(1-f)$ uev $(1-f)$. Similar to Theorem 2.2, we have $a+(1-a) b z \in U^{-}(I)$, where $z=y s(v(1-f)(1+f u e v(1-f))-a)$. By Lemma 4.1, we have $z \in R$ such that $x+(1+z(1-a)) b=x+b+z(1-a) b \in U^{-}(I)$. Applying Lemma 4.2 to $a x+b=1$, we get some $w \in R$ such that $a+b w \in U^{-}(I)$. We easily check that $w \in I$, as asserted.

Theorem 4.4. Let $I$ be an exchange ideal of a ring $R$. If for any regular $x \in 1+I$ there exists $u \in U^{-}(I)$ such that $x=x u x$, then $I$ is locally comparable.

Proof. Suppose that $a x+b=1$ with $a, x \in I, b \in R$. Then $(a+b) x+b(1-x)=$ $1, a+b \in 1+I, x \in I$ and $b(1-x) \in 1+I$. In view of Lemma 4.3, we have $y \in R$ such that $a+b(1+(1-x) y)=a+b+b(1-x) y \in U^{-}(I)$. Clearly, $u \in 1+I$, and so $u \in U(I)$. Therefore $I$ is a locally comparable ideal by Corollary 2.5 .

Recall that an ideal $I$ of a ring $R$ is a $B$-ideal, provided that $a R+b R=R$ with $a \in 1+I$ and $b \in R$ implies that there exists $y \in R$ such that $a+b y \in U(R)$, where $U(R)$ is the group of units in $R$ (cf. [5]). As an immediate consequence, we derive

Corollary 4.5. Every exchange B-ideal is locally comparable. 
Theorem 4.6. Every exchange ideal satisfying the comparability axiom is locally comparable.

Proof. Given any regular $x \in 1+I$, there exists $y \in 1+I$ such that $x=x y x$. So $1-y x, 1-x y \in I$. Hence either $(1-x y) R \lesssim^{\oplus}(1-y x) R$ or $(1-y x) R \lesssim^{\oplus}(1-x y) R$. If $(1-x y) R \lesssim^{\oplus}(1-y x) R$, we have an injection $\psi:(1-x y) R \rightarrow(1-y x) R$. From $R=y x R \oplus(1-y x) R=x y R \oplus(1-x y) R$ and $\phi: x y R=x R \cong y x R$, we have $u \in \operatorname{End}_{R}(R)$ so that $u$ restricts to $\phi$ and $u$ restricts to $\psi$. Then $x=x u x$ with left invertible $u \in R$. If $(1-y x) R \lesssim^{\oplus}(1-x y) R$, analogously, we derive that $x=x u x$ for a right invertible $u \in R$. Consequently, $x=x u x$ for a $u \in U^{-}(R)$. Therefore we complete the proof by Theorem 4.4.

Corollary 4.7. Let I be an exchange ideal satisfying the comparability axiom. Then every square regular matrix over I admits a diagonal reduction.

Proof. It follows by Theorem 4.6 and Theorem 3.5.

Acknowledgement. The authors would like to thank the referee for the valuable suggestions and comments.

\section{References}

[1] P. Ara, Extensions of exchange rings, J. Algebra, 197(2) (1997), 409-423.

[2] H. Chen, Elements in one-sided unit regular rings, Comm. Algebra, 25(8) (1997), 2517-2529.

[3] H. Chen, On generalized stable ideals, Comm. Algebra, 38(10) (2010), 35673579 .

[4] H. Chen, On quasi-stable exchange ideals, J. Korean Math. Soc., 47(1) (2010), $1-15$.

[5] H. Chen, Rings Related to Stable Range Conditions, Series in Algebra, 11, World Scientific Publishing Co. Pte. Ltd., Hackensack, NJ, 2011.

[6] C. Huang, Refinement rings, exchange property and comparability, Bull. Korean Math. Soc., 48(3) (2011), 455-468.

[7] D. Khurana, T. Y. Lam and P. P. Nielsen, Exchange elements in rings, and the equation $X A-B X=I$, Trans. Amer. Math. Soc., 369(1) (2017), 495-516.

[8] F. Perera, Lifting units modulo exchange ideals and $C^{*}$-algebras with real rank zero, J. Reine Angew. Math., 522 (2000), 51-62. 
Handan Kose (Corresponding Author)

Department of Mathematics

Kirsehir Ahi Evran University

40100 Kirsehir, Turkey

e-mail: handan.kose@ahievran.edu.tr

Yosum Kurtulmaz

Department of Mathematics

Bilkent University

Ankara, Turkey

e-mail: yosum@fen.bilkent.edu.tr

\section{Huanyin Chen}

Department of Mathematics

Hangzhou Normal University

Hangzhou, China

e-mail: huanyinchen@aliyun.com 